\title{
Melting and solidification of lead nanoparticles embedded in Aluminium-Gallium matrices
}

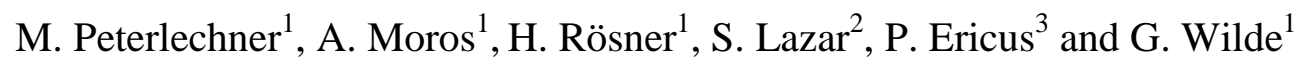

${ }^{1}$ Institut für Materialphysik, Universität Münster, Wilhelm-Klemm-Straße 10, D-48149 Münster, Germany

${ }^{2}$ FEI Company, Achtseweg Noord 5, 5600 KA Eindhoven, The Netherlands

${ }^{3}$ National Center for Electron Microscopy (NCEM), Lawrence Berkeley National Laboratory, One Cyclotron Road, MS 72, Berkeley, CA 94720

\begin{abstract}
Analyzing the impact of the host matrix on the melting/solidification of embedded $\mathrm{Pb}$ nanoparticles, various $\mathrm{Ga}$ concentrations $(0,1,3$ and 6 at.\%) were added to the Al matrix within the solid solution regime to modify the mismatch between matrix and nanoparticles by expanding the host lattice. Using transmission electron microscopy and differential scanning calorimetry, a strong impact of an atomically flat Ga-enriched wetting layer around the $\mathrm{Pb}$ nanoparticles on the melting/solidification was observed. The Ga-enrichment was found to be about $\sim 3 \%$ above the matrix level, independent of the Ga concentration in the matrix. It is concluded that upon solidification vacancies arising from the $3.3 \%$ volume shrinkage of liquid $\mathrm{Pb}$ are filled by the fast diffusing $\mathrm{Ga}$. The Ga-enriched layer chemically and mechanically decouples the matrix from the nanoparticles leading to a more freestanding particle behaviour showing strong undercooling upon solidification and reduced or no superheating upon melting.
\end{abstract}

\section{Keywords}

nanoparticles; melting; transmission electron microscopy; EDX; interface; 


\section{Introduction}

Nanoparticles embedded in a matrix with mutually immiscible constituents offer ideal conditions for studying the impact of hetero-interfaces on reversible phase transitions. Al-Pb composites consisting of nanometer-sized $\mathrm{Pb}$ inclusions embedded in a polycrystalline $\mathrm{Al}$ matrix are a model system for size-dependent melting studies [1-6]. A local minimum free-energy configuration is obtained when the nanoparticles with cube-octahedral shape adopt a cube-oncube orientation relationship with the matrix, having larger $\{111\}$ - and smaller $\{100\}$-facets [1-3,7-13]. The metal/metal interfaces were found to maintain a strict cube-on-cube orientation relationship although exhibiting a large lattice mismatch of $22 \%$ [1-3,7-9]. To explain the relationship between morphology and strain, an elastic accommodation of the lattice mismatch via vacancies was claimed [8,9]. Transmission electron microscopy (TEM) and X-ray diffraction (XRD) in-situ heating experiments have shown that the $\mathrm{Pb}$ melting temperature $T_{\mathrm{m}}$ of such particles can be increased by more than $60 \mathrm{~K}$ with respect to the bulk melting point of $\mathrm{Pb}[1-3,8,11,14,15]$. The observation of an elevated melting point of nanoparticles was correlated with faceted particle shapes, semi-coherent interfaces and pressure, whereas melting point depressions were attributed to non-faceted particle shapes [2,3,7,16,17]. It has been shown that the melting point of non-faceted $\mathrm{Pb}$ inclusions embedded in $\mathrm{Al}$ can be manipulated by an appropriate heat treatment changing the particle morphology from non-faceted to faceted shapes which then increases the melting point from below to above the bulk melting of $\mathrm{Pb}[7,18]$. Such melting behaviour motivates an interest in the relation between strains and interfacial morphology. The elastic strain caused by melting and solidification of small $\mathrm{Pb}$ inclusions in Al has been monitored by in-situ TEM [19]. When the nanoparticle is liquid, the surrounding Al matrix is free of strain. However, as the inclusion solidifies it contracts causing elastic distortions in the $\mathrm{Al}$ matrix, which are thought to be relieved by lattice vacancies [19]. Evidence of localized misfit via geometrically necessary dislocations has also been found [7,20,21], and the nanoparticles show reduced melting enthalpy [22]. Moreover, liquid 
$\mathrm{Pb}$ inclusions showing Brownian motion in three dimensions have been observed [23,24]. It has been shown that the motion of the $\mathrm{Pb}$ inclusions is controlled by volume vacancy diffusion [24].

The goal of this study is to investigate the impact of the host matrix on the melting and solidification behavior of the $\mathrm{Pb}$ nanoparticles. For this purpose, $\mathrm{Ga}$ was added to the matrix within the solid solution regime. Alloying of Ga expands the lattice of the Al matrix and thus reduces the mismatch between matrix and $\mathrm{Pb}$ particle. Moreover, $\mathrm{Ga}$ is immiscible with $\mathrm{Pb}[25]$ and thus the melting and solidification of the $\mathrm{Pb}$ nanoparticles was expected to be affected by the $\mathrm{Ga}$ addition without involving changes in the composition of the $\mathrm{Pb}$ nanoparticles. If the hypothesis of strain relief via accumulated lattice vacancies upon solidification holds, and the accumulated lattice vacancies required for the observed Brownian motion of the liquid $\mathrm{Pb}$ nanoparticles are still present, then the Ga addition is expected to show a segregation effect around the $\mathrm{Pb}$ nanoparticle interfaces. The present study demonstrates this. The solidification was drastically influenced by the Ga addition leading to an undercooling of more than $100 \mathrm{~K}$ for $\mathrm{Al}_{93} \mathrm{Ga}_{6} \mathrm{~Pb}_{1}$. Moreover, Ga segregation was found around the $\mathrm{Pb}$ nanoparticles, which has been shown to be independent of the nominal Ga (1-6 at.\%) addition and amounts to about 3 at. $\%$ above the matrix level, thus accommodating the volume jump of $3.3 \%$ of $\mathrm{Pb}$ during solidification.

\section{Experimental}

Samples of $\mathrm{Al}_{99-\mathrm{x}} \mathrm{Ga}_{\mathrm{x}} \mathrm{Pb}_{1}$ composites (with $\mathrm{x}=0,1,3$ and 6; all in at.\%) within the solid solution regime were produced by melt spinning. Initially, ingots of approximately $3 \mathrm{~g}$ were prepared by arc melting under protective Ar gas atmosphere using high-purity $\mathrm{Al}(5 \mathrm{~N}), \mathrm{Ga}(5 \mathrm{~N})$ and $\mathrm{Pb}(5 \mathrm{~N})$. The ingots were then melted in a quartz glass crucible by induction melting under a low pressure Ar atmosphere. The ejection of the melt onto a rotating copper wheel (wheel-surface velocity of $31 \mathrm{~m} / \mathrm{s}$ ) resulted in crystalline ribbons with thicknesses of 50-60 
$\mu \mathrm{m}$. As-cast samples were analyzed by TEM and EDX directly after processing, without any heating where the nanoparticles get molten and re-solidified. The chemical composition after rapid solidification was verified by energy dispersive X-ray (EDX) analysis in a scanning electron microscope (FEI Nova Nano SEM 230) and corresponds to the nominal composition (see Tab.1). For all EDX measurements the ZAF method was applied, without using standards. Calorimetric measurements using a power-compensated differential scanning calorimeter (Diamond DSC, Perkin Elmer) under constant Ar flow were performed using a constant heating and cooling rate of $20 \mathrm{~K} / \mathrm{min}$. Sample masses were typically around $30 \mathrm{mg}$. According to Vegards Law the lattice constant of $\mathrm{Al}(\mathrm{Ga})$ can be given for pure $\mathrm{Al}$, the $\mathrm{Al}-1$ at.\% $\mathrm{Ga}$, Al-3at.\%Ga, Al-6at.\%Ga to be $4.0496 \AA$, $4.0543 \AA$, $4.0638 \AA$ and $4.0780 \AA$, respectively. Consequently the mismatch of $\mathrm{Al}(\mathrm{Ga})$ to $\mathrm{Pb}$ can be given for pure $\mathrm{Al}$, the $\mathrm{Al}-1$ at.\% $\mathrm{Ga}, \mathrm{Al}-$ 3at.\%Ga, Al-6at.\%Ga to be $22.2 \%, 22.1 \%, 21.9 \%$ and $21.5 \%$, respectively.

TEM specimens for analytical STEM were initially mechanically polished with $5 \mu \mathrm{m} \mathrm{SiC}$ and subsequently ion-milled at liquid nitrogen temperatures using the PIPS II (Gatan PIPS 695) with acceleration voltages from $3.0-0.5 \mathrm{kV}$ and incident beam angles of $4^{\circ}$ for top and bottom. STEM imaging and chemical analyses were performed using a double-corrected, monochromated FEI Temis Titan 60-300 operated in STEM mode at $300 \mathrm{kV}$ using the ChemiSTEM technology [26,27] (X-FEG and Super-X detector). Additionally, high-angle annular darkfield (HAADF) STEM images were acquired using the TEAM 0.5 instrument at the National Center for Electron Microscopy facility in the Molecular Foundry (MF, Berkeley).

\section{Results}

\section{Microstructure}

Rapid solidification results in a bimodal size distribution of faceted $\mathrm{Pb}$ nanoparticles dispersed within Al-Ga matrices, as it was reported for the AlPb composite [22] and turned out 
to be independent of the Ga content. Larger $\mathrm{Pb}$ inclusions with curved interfaces are generally found at grain boundaries and at the sample surface, while $\mathrm{Pb}$ nanoparticles in the grain interior are faceted displaying a Wulff-shape of truncated cube-octahedrons, having diameters from $5 \mathrm{~nm}$ to $200 \mathrm{~nm}$ in size. Such $\mathrm{Pb}$ nanoparticles exhibit a cube-on-cube orientation relationship to the Al-Ga matrix, independent of the amount of Ga added.

\section{Calorimetry (DSC)}

The melting and solidification transitions of different $\mathrm{Al}_{99-\mathrm{x}} \mathrm{Ga}_{\mathrm{x}} \mathrm{Pb}_{1}$ composites (with $\mathrm{x}=0,1$, 3 and 6) have been systematically investigated by differential scanning calorimetry (DSC). The results are shown in Fig.1 and summarized in Tab.2.

Without $\mathrm{Ga}$, melting of the $\mathrm{Pb}$ nanoparticles occurs partially at the bulk melting temperature, indicated by a dashed line in Fig.1, and partially at higher temperatures where three additional and overlapping peaks are detected. The melting signal extends over a range of $40 \mathrm{~K}$. With increasing $\mathrm{Ga}$ content the superheating of $\mathrm{Pb}$ particles is reduced. Finally, at a $\mathrm{Ga}$ concentration of 6 at.\%, the $\mathrm{Pb}$ melting signal is detected predominately in a single peak about $7 \mathrm{~K}$ below the bulk melting temperature.

Similar, upon cooling solidification occurs at monotonously reduced temperatures with increasing Ga content. The melting and solidification onset temperatures are plotted in Fig. 2 as a function of the Ga content. The melting onset is decreased by $7 \mathrm{~K}$ and the solidification onset temperature decreases by $103 \mathrm{~K}$.

\section{Chemical Analyses (EDX)}

EDX analyses were carried out on individual $\mathrm{Pb}$ nanoparticles for various $\mathrm{Ga}$ concentrations. The $\mathrm{Pb}$ nanoparticles were mostly oriented along the $\langle 110\rangle$-zone axis of the fcc matrix $\mathrm{Al}$ and imaged using HAADF-STEM, see Figs. 3a and 4a (note: white dots are Moiré pattern due to the overlap of $\mathrm{Al}$ and $\mathrm{Pb}$ atomic columns in projection). Thus, 6 facets (four $\{111\}$ and two 
$\{100\}$ facets $)$ are imaged in edge-on conditions. The results of the chemical analyses using a spot size of $\sim 1 \AA$, representing 3 and 6 at.\% Ga, are shown in the Figs. $3 b$-e and 4b-e. The chemical analyses reveal that Ga segregates at the particle-matrix hetero-interface (Figs. 3d and $4 \mathrm{~d})$. The Ga concentration has been traced in the form of profiles across the $\mathrm{Pb}$ nanoparticle (see boxed area in Figs. 3d and 4d). They show peaks in the Ga signal at the interfaces (Figs. $3 \mathrm{e}$ and $4 \mathrm{e}$ ). The increment, which is defined as the difference between the Ga concentration in the matrix and the peak value at the interface, is determined to be $\sim 3 \%$ (2.6 at. $\% \pm$ $0.3 \%$ on average for four $\mathrm{Pb}$ nanoparticles in $\mathrm{Al}$-matrices with different $\mathrm{Ga}$ concentrations). It must be noted, that this evaluation does not take into account the projected thickness of embedded interfaces and the overlying/underlying matrix; thus, the absolute concentration at the interface is equal or higher than 2.6 at.\%. However, all measurements were performed at areas as thin as possible and the evaluated Ga content at the interface is independent of the matrix concentration of $\mathrm{Ga}$. The Ga-concentration level across the Pb nanoparticle is always higher on average than the surrounding matrix concentration due to the projected overlap of the other eight remaining Ga-enriched interfaces, which are not imaged in edge-on conditions. The width of all the Ga signal at the $\{111\}$ interfaces is always less than $2.6 \mathrm{~nm}$, and independent of the Ga content of the matrix. It should be noted, that STEM image simulations (not shown here) indicate an EDX signal broadening at the interface of $0.5 \mathrm{~nm}$ for a $10 \mathrm{~nm}$ particle in a 20 $\mathrm{nm}$ thin sample. In the present experiments the signal broadening is estimated by the EDX signal broadening of $\mathrm{Al}$ and $\mathrm{Pb}$ at the interface, which is always below $2.1 \mathrm{~nm}$. The selected EDX map in Fig. 3 shows Ga interfaces with a width of less than $1.5 \mathrm{~nm}$, and a signal broadening determined from the width of the $\mathrm{Al}$ and $\mathrm{Pb}$ signals at the interface of about $1 \mathrm{~nm}$. Similar, the measured interface width of the Ga signal in Fig. 4 is about $2.1 \mathrm{~nm}$, and the interface width of the $\mathrm{Al} / \mathrm{Pb}$ signals about $1.7 \mathrm{~nm}$. Thus, the Ga layer thickness from which the main contribution to the Ga EDX signal originates is around $0.5 \mathrm{~nm}$, which amounts basically to one atomic layer. 
These analyses are focused on the $\{111\}$-interfaces. It should be noted that the $\{100\}$ interfaces show a lower Ga enrichment (roughly the half) than the $\{111\}$-ones. This is a result of the measurement projection: the $\{111\}$-interfaces are observed to be typically twice as long as the $\{100\}$-interfaces. Thus, the actual Ga enrichment at the $\{100\}$ interfaces is therefore, within the measurement accuracy, in agreement with that of the $\{111\}$ interfaces.

\section{High-resolution HAADF-STEM}

An independent evaluation of the Ga enrichment can be achieved by the comparison of the intensities of individual atomic columns in a high-resolution HAADF-STEM image and was applied on a representative $\mathrm{Pb}$ nanoparticle (see Fig. 5). The intensities in the atomic layers around a Pb nanoparticle (line profile over the red box in Fig. 5a, shown in Fig. 5b) have been compared with the intensity of a reference area in the matrix (line profile taken from the yellow marked box in Fig. 5a). The measured results are summarized in Tab.3. Since this method probes in first order the change in the average atomic number $\mathrm{Z}$, a qualitative comparison with the chemical composition deduced by EDX can be made. The change in the HAADF signal intensity (using $\mathrm{I} \sim \mathrm{Z}^{1.7}$, neglecting channeling effects at the interface) is in agreement with a mean $\mathrm{Ga}$ enrichment of 3.3 at.\% above matrix level for the analysed $\{111\}$-interface. It may be noted, that the Ga enrichment at the interface never exceeded the equilibrium bulk saturation concentration of 9 at.\% $\mathrm{Ga}$ in $\mathrm{Al}[25]$. Figure $5 \mathrm{~b}$ shows the intensity profile across a $\{111\}$-interface. The detectable interface width is 1 atomic layer, which is in agreement with the beam-broadening corrected signal detected by EDX.

\section{Discussion}

The results of the experimental data are discussed in the following with regard to pressure/strain, interface and layering effects. 


\section{1) Pressure and strain effects}

Different species of embedded $\mathrm{Pb}$ particles in the $\mathrm{Al}$ matrix show different contributions to the DSC signal upon heating, as shown in Fig. 1. Besides larger $\mathrm{Pb}$ particles at the sample surface and at grain boundaries (GBs), nanoparticles at different types of sites are present. In contrast to heating, upon cooling only a single peak occurs in the DSC signal. This indicates that the solidification kinetics are similar for smaller and larger particles as well as for $\mathrm{Pb}$ particles located at different sites (GBs, surfaces, grain interiors). Since nanoparticles located at surfaces and grain boundaries have the possibility of pressure relief, it can be excluded that pressure plays a major role in the solidification process. This is in agreement with recent $\mathrm{X}$ ray diffraction strain measurements of ion-implanted $\mathrm{Pb}$ nanoparticles in an $\mathrm{Al}$-matrix, where basically no strain or pressure on the nanoparticles was observed [28]. During solidification $\mathrm{Pb}$ undergoes a volume contraction of $3.3 \%$ leading to a negative pressure on the nanoparticles in the solid state [19]. This relative pressure contribution to the enthalpy is different for different $\mathrm{Pb}$ particle sizes and it is site-dependent. For the case of $\mathrm{Pb}$ nanoparticles embedded in an Al matrix a vacancy controlled stress relaxation process was reported [19].

The observed change of the melting onset temperature is often explained by the ClausiusClapeyron equation; the present DSC data including the melting onset temperature of bulk $\mathrm{Pb}$ are summarized in Table 2. It should be noted that besides the melting temperature also the melting enthalpy is reduced in the case of the melting of $\mathrm{Pb}$ nanoparticles in an Al-matrix [9]. Moreover, an increased melting temperature is in agreement with a positive pressure on the particles, in contrast to the volume contraction upon solidification. Consequently, a full description of the present results just by the Clausius-Clapeyron equation is not possible. Thus, the interface contribution remains crucial for the melting and solidification behavior, which is not taken into account by the Clausius-Clapeyron equation. 
It was derived earlier that freestanding nanoparticles show a melting point reduction, whereas embedded nanoparticles can show increased melting temperatures due to a hydrostatic pressure and/or a stable interface configuration [6]. The degree of coherency affects the interface energy, and thus the enthalpy difference between the solid and liquid states. It has been shown that $\mathrm{Pb}$ nanoparticles have semi-coherent interfaces with misfit dislocations [20,21]. Therefore, to minimize the interface energy, the size distribution of nanoparticles is modulated by a multiplicity of the spacing of misfit dislocations, leading to so called magic sizes [9]. The Gaenriched matrix reduces the misfit compared to a pure $\mathrm{Al}$ matrix and consequently reduces the misfit strain at the interfaces and the corresponding interface energies. Accordingly, one might expect an increase of the superheating due to a more stable interface with increasing $\mathrm{Ga}$ content, which is not in agreement with the experimental findings. Thus, we conclude that the Ga segregation leads to a wetting layer wrapping the $\mathrm{Pb}$ particle, and this layer mechanically decouples the $\mathrm{Pb}$ nanoparticles which then displays a melting/solidification behavior more similar to free-standing particles.

\section{2) Interface effect}

In agreement with the conclusion above, the local STEM EDX analysis (Fig.3 and Fig.4) shows that a Ga enrichment occurs locally at the interfaces between $\mathrm{Pb}$ and $\mathrm{Al}$. Based on the interpretation of the signal broadening of the STEM EDX signals of $\mathrm{Al}$ and $\mathrm{Pb}$, it can be concluded that the Ga enrichment is basically confined to a single atomic layer, in agreement with the HAADF STEM image intensity evaluation. The enrichment of the Ga is determined to be 2.6 at.\% by STEM EDX and estimated to be 3.3 at.\% by HAADF STEM, independent of the overall $\mathrm{Ga}$ concentration in the matrix. It has been claimed that lattice vacancies are a key in accommodating the misfit strain upon solidification of nanoparticles embedded in $\mathrm{Al}$ $[8,9,24]$. Based on this assumption, an increased number of vacancies should be available 
around the $\mathrm{Pb}$ nanoparticles leading then to an accelerated $\mathrm{Ga}$ diffusion into this region and thus to the observed $\mathrm{Ga}$ enrichment. Taking this scenario at face value, the corresponding increment of the excess volume of the Ga concentration would then correspond to the total excess volume present as lattice vacancies around the $\mathrm{Pb}$ nanoparticles now filled with $\mathrm{Ga}$ atoms, yielding about $3 \%$. It is noted, that the volume jump of $\mathrm{Pb}$ from liquid to solid is $3.3 \%$ [19].

This estimation of the vacancy concentration is influenced by the change of the chemical potential around the nanoparticles, and by the error of the measurement which is mainly caused by dynamic scattering effects of electrons and modified scattering cross sections at the interface. $\mathrm{Ga}$ and $\mathrm{Pb}$ are completely immiscible, thus it is expected that there is basically no chemical driving force for diffusion of $\mathrm{Ga}$ into the $\mathrm{Al}-\mathrm{Pb}$ interface. It was reported earlier, that Ga segregation to grain boundaries in pure Al shows only small segregation enthalpies (190 $\mathrm{meV}$ in $\mathrm{Al}-2 \mathrm{at} . \% \mathrm{Ga}$ ) [29], which is dependent on the matrix concentration. In the present work, the increment defined as the difference between the Ga concentration in the matrix and the Ga peak value at the interface was observed to be basically independent of the Ga content of the matrix, and thus, segregation effects are not pronounced. Consequently, the $3 \%$ of excess vacancy concentration can be seen as a good estimation. In agreement with that, the width of the Ga-enriched zone was observed to be the same for different Ga contents, basically a single atomic layer.

The addition of Ga changes the interface (stress and chemical potential) and thus the interface energy. Consequently, upon cooling the barrier for heterogeneous nucleation of solid $\mathrm{Pb}$ at the $\mathrm{Al}$ interface is higher, since with increasing Ga content the possible amount of undercooling increases. According to the classical nucleation theory for heterogeneous nucleation, the nucleation frequency, I, can be written as:

$$
\mathrm{I}=\mathrm{A} \exp \left[-\mathrm{B} /\left(\left(\mathrm{T}_{\mathrm{m}}-\mathrm{T}\right)^{2} \cdot \mathrm{T}\right)\right]
$$


where $A=N_{c}(k T / h) \exp (-Q / k T)$ and $B=K \sigma^{3} T^{3}{ }_{m} f(\Theta) / k L^{2}$. Here, $N_{c}$ is the number of catalytic nuclei, $\mathrm{k}$ the Boltzmann-constant, $\mathrm{h}$ the Planck-constant, $\mathrm{Q}$ the activation energy for a $\mathrm{Pb}$ atom to jump across the solid-liquid interface, $\mathrm{K}=16 \pi / 3$ the shape factor, and $\sigma$ the solidliquid interface energy of $\mathrm{Pb}-\mathrm{Pb} . \mathrm{L}$ is the latent heat of melting, the melting temperature is $\mathrm{T}_{\mathrm{m}}$ and $\mathrm{T}$ is the temperature. The contact angle $\Theta$ is used in the geometrical function, which is defined as $\mathrm{f}(\Theta)=2-3 \cos (\Theta)+\cos ^{3}(\Theta / 4)$. Based on that, at the point of maximum rate the following relation can be deduced [30]:

$$
\text { Bं }\left(3 \mathrm{~T}_{\max }-\mathrm{T}_{\mathrm{m}}\right) / \mathrm{A}\left(\mathrm{T}_{\mathrm{m}}-\mathrm{T}_{\max }\right)^{3} \mathrm{~T}^{2}{ }_{\max }=\exp \left(-\mathrm{B} /\left(\mathrm{T}_{\mathrm{m}}-\mathrm{T}_{\max }\right)^{2} \mathrm{~T}_{\max }\right) \quad \text { Eq. }(2)
$$

The constants A and B can be determined from the plot given in Fig. 6. The slope corresponds to $-\mathrm{B}$ and the $\mathrm{y}$-intercept corresponds to $\ln (\mathrm{A} / \mathrm{B})$. The linear fits in Fig. 6 show reasonably good agreement with the experimental data. Using the constant B, the contact angle and consequently the interface energy of the $\mathrm{Al}-\mathrm{Pb}$ with $\mathrm{Ga}$ can be determined, as listed in Table 4 . For that, $\cos (\Theta)=\left(\sigma_{L}-\sigma_{S}\right) / \sigma$ was applied, where $\sigma_{L}-\sigma_{S}$ is the difference between the interface energy of liquid-Pb/solid-Al and solid- $\mathrm{Pb} /$ solid-Al, a relative interface energy. This relative interface energy decreases with increasing Ga concentration. Therefore, the driving force for solidification (to form a solid-solid interface) is reduced with increasing Ga concentration, yielding a large undercooling for high Ga concentrations. With increasing Ga content the melting enthalpy increases, as shown in Table 2. This can be explained by the fact that the energetically "costly" liquid-Pb/solid-Al is gradually less "expensive" with increasing Ga content.

\section{3) Layering and transformation kinetics}

Phase transformations in confined systems, where the confinement involves interfaces of the transforming nanoscale material with a crystalline confining structure, need to regard the im- 
pact of so-called layering effects that affect the atomic structure of the transforming material near the interface via the long-range interatomic interaction potential of the confinement structure. Measurements by X-ray diffraction on liquid $\mathrm{Pb}$ [31] or experimental analyses of a sapphire/liquid-Al [32] by in-situ HR-TEM have shown clear structural evidence that the vicinity of the interface of the crystalline containment induces enhanced order in the liquid that is measurable over about four to five average interatomic distances. Moreover, layer ordering in embedded liquid nanoparticles of $\mathrm{Xe}$ in $\mathrm{Al}$ showed ordering of up to three atomic layers at the interface [33]. DSC results on the melting transition of $\mathrm{Pb}$ in $\mathrm{Al}$ [22] indicated that layering is also significant for describing the melting / solidification transformations of the system analysed in this work.

Layering, i.e. enhanced structural order, in a liquid causes not only a reduction in the transformation enthalpy between liquid and crystal, thereby modifying the thermodynamics, but also has an important impact on the kinetics of the transformations that are initiated by nucleation. Particularly for elemental metallic melts that solidify to densely packed crystalline structures (such as $\mathrm{Pb}$ ), the presence of crystal-like precursor structures in the undercooled liquid state severely limits the achievable undercooling level, since the transformation can be initiated only by minor local atomic shuffling events. In the current work, this behavior is reflected by the low undercooling of $\mathrm{Al}_{99} \mathrm{~Pb}_{1}$ of only about $4 \mathrm{~K}$. Figure 2 clearly shows a drastic increase of the undercooling prior to solidification with increasing Ga content. Since the Ga in solid solution in the $\mathrm{Al}$ matrix serves to minimize the mismatch between the lattices of $\mathrm{Al}(\mathrm{Ga})$ and $\mathrm{Pb}$, it is only the Ga-enriched interface layer that causes the undercooling enhancement by increasing the nucleation barrier. This observation can be interpreted as a reduction of the ordering of the liquid $\mathrm{Pb}$ near the particle-matrix interface due to the Gaenriched layer. In fact, this interpretation leads to the expectation that an enhanced absolute concentration of $\mathrm{Ga}$ in the interface layer should linearly decrease the effective area where layering occurs. A decrease of the volume affected by layering follows a decrease of the nu- 
cleation sites. Thus, since to a first order approximation the partial volume of the liquid particle affected by layering scales with the area of the particle matrix interface that causes the layering, a linear dependence of the undercooling on the absolute Ga concentration might be expected and is basically found experimentally.

At the same time, the melting behavior also changes systematically with increasing Ga addition: the characteristic temperatures of the individual melting maxima decrease and finally only one peak remains that shows an onset slightly below the melting temperature of bulk $\mathrm{Pb}$. This behavior indicates that the presence of Ga efficiently decouples the nucleation of melting from the specific local atomic configuration, i.e. Ga particles situated in grain interiors, at dislocation cores or at matrix grain boundaries melt at similar temperatures.

Thus, it appears that the "wrapping" of the nanoparticles by a Ga-enriched layer decouples the particles from the interatomic interaction potential of the $\mathrm{Al}(\mathrm{Ga})$ matrix. This result is rather unexpected and astonishing, since it should be kept in mind that the thickness of the Gaenriched layer amounts to only one monolayer. Thus, one monolayer of Ga-enriched $\mathrm{Al}(\mathrm{Ga})$ apparently serves as a disordered confinement that allows observing nanoparticle properties and behavior comparable to free nanoparticles. This rather unexpected result might also have bearings concerning the possibilities for functional nanostructuring, since the present results indicate the opportunity for effective containments and boundaries with mono-atomic dimensions.

\section{Conclusions}

A deep undercooling of up to $103 \mathrm{~K}$ was found for the solidification of embedded $\mathrm{Pb}$ nanoparticles in the $\mathrm{Al}$ matrix due to $\mathrm{Ga}$ addition in the range of 1 to 6 at.\%. A Ga enriched atomically flat layer around the $\mathrm{Pb}$ nanoparticles yields a constant $\mathrm{Ga}$ enrichment of about 3 
$\%$, independent of the overall $\mathrm{Ga}$ concentration in the matrix. Since $\mathrm{Ga}$ and $\mathrm{Pb}$ are completely immiscible, we conclude that the Ga enriched layers at the Al-Pb interface form by filling an increased number of vacancies present around the $\mathrm{Pb}$ nanoparticles arising during solidification due to the $3.3 \%$ volume jump between the liquid and the solid state of $\mathrm{Pb}$. Such zones are interpreted as buffer zones between the $\mathrm{Al}(\mathrm{Ga})$ matrix and the $\mathrm{Pb}$ nanoparticle impeding the nucleation of the liquid $\mathrm{Pb}$ due to their chemical and mechanical decoupling and thus leading to the deep undercooling upon solidification and a reduced overheating upon melting.

\section{Acknowledgment}

We kindly acknowledge financial support by the DFG via WI 1899/14-1. We thank Dr P. Schlossmacher (FEI) for enabling the STEM EDX measurements at the Nanoport in Eindhoven. We are grateful to Dr M. Falke (Bruker Nano $\mathrm{GmbH}$ ) for providing access to Quantax software. High-resolution STEM imaging was accomplished using the Molecular Foundry (Lawrence Berkeley Lab, which is supported by the U.S. Department of Energy under Contract \#DE-AC02-05CH1123). 


\section{References}

[1] K.I.K. Moore, K. Chattopadhyay, B. Cantor, In situ transmission electron microscope measurements of solid $\mathrm{Al}$-solid $\mathrm{Pb}$ and -solid Al-liquid $\mathrm{Pb}$ surface-energy anisotropy in rapidly solidified Al-5 \% Pb ( by mass ), Proc. R. Soc. London. A. Math. Phys. Sci. 414 (1987) 499-507. http://www.jstor.org/stable/2398087 (accessed May 8, 2011).

[2] K.I. Moore, L.D. Zahng, B. Cantor, Solidification of Pb Particles embedded in al, Acta Metall. Mater. 38 (1990) 1327-1342.

[3] D.L. Zhang, B. Cantor, Melting behaviour of In and $\mathrm{Pb}$ particles embedded in an $\mathrm{Al}$ matrix, Acta Metall. Mater. 39 (1991) 1595-1602. doi:10.1016/0956-7151(91)90247$\mathrm{X}$.

[4] K. Chattopadhyay, R. Goswami, Melting and superheating of metals and alloys, Prog. Mater. Sci. 42 (1997) 287-300. doi:10.1016/S0079-6425(97)00030-3.

[5] E. Johnson, H.H. Andersen, U. Dahmen, Nanoscale lead and noble gas inclusions in aluminum: structures and properties., Microsc. Res. Tech. 64 (2004) 356-72. doi:10.1002/jemt.20097.

[6] Q.S. Mei, K. Lu, Melting and superheating of crystalline solids: From bulk to nanocrystals, Prog. Mater. Sci. 52 (2007) 1175-1262.

doi:10.1016/j.pmatsci.2007.01.001.

[7] H. Rösner, P. Scheer, J. Weissmüller, G. Wilde, The impact of particle morphology on the melting characteristics of matrix-embedded $\mathrm{Pb}$ nanocrystals, Philos. ... 83 (2003) 511-523. doi:10.1080/0950083031000140943.

[8] H. Gabrisch, L. Kjeldgaard, E. Johnson, U. Dahmen, Equilibrium shape and interface roughening of small liquid $\mathrm{Pb}$ inclusions in solid Al, Acta Mater. 49 (2001) 42594269. doi:10.1016/S1359-6454(01)00307-X.

[9] U. Dahmen, S. Xiao, S. Paciornik, E. Johnson, A. Johansen, Magic-Size Equilibrium Shapes of Nanoscale Pb Inclusions in Al, Phys. Rev. Lett. 78 (1997) 471-474. doi:10.1103/PhysRevLett.78.471.

[10] P. a. Thackery, R.S. Nelson, The formation of precipitate phases in aluminium by ion implantation, Philos. Mag. 19 (1969) 169-180. doi:10.1080/14786436908217771.

[11] H.W. Sheng, G. Ren, L.M. Peng, Z.Q. Hu, K. Lu, Superheating and melting-point depression of $\mathrm{Pb}$ nanoparticles embedded in Al matrices, Philos. Mag. Lett. 73 (1996) 
$179-186$.

http://www.tandfonline.com/doi/abs/10.1080/095008396180812 Inpapers2://publication /doi/10.1080/095008396180812 \nhttp://www.informaworld.com/openurl?genre=article $\&$ doi $=10.1080 / 095008396180812 \&$ magic $=$ crossref $\|$ D404A21C5BB053405B1A640AF FD44AE3.

[12] E. Johnson, A. Johansen, U. Dahmen, S. Chen, T. Fujii, Shapes and sizes of nanoscale $\mathrm{Pb}$ inclusions in Al, Mater. Sci. ... 304-306 (2001) 187-193. doi:10.1016/S09215093(00)01462-3.

[13] A. Landa, P. Wynblatt, E. Johnson, U. Dahmen, Computer simulation of Pb/Al interfaces, Acta Mater. 48 (2000) 2557-2563. doi:10.1016/S1359-6454(00)00059-8.

[14] L. Grabaek, J. Bohr, E. Johnson, A. Johansen, L. Sarholt-Kristensen, H.H. Andersen, Superheating and Supercooling of Lead Precipitates in Aluminum, Phys. Rev. Lett. 64 (1990) 934-937.

[15] H. Sheng, K. Lu, E. Ma, Melting of embedded Pb nanoparticles monitored using hightemperature in situ XRD, Nanostructured Mater. 10 (1998) 865-873. doi:10.1016/S0965-9773(98)00122-6.

[16] H. Rösner, T. Scherer, G. Wilde, Electron tomography of lead nano-inclusions in aluminium, Scr. Mater. 60 (2009) 168-170. doi:10.1016/j.scriptamat.2008.09.025.

[17] A.K. Malhotra, D.C. Van Aken, On the effect of matrix relaxation during the melting of embedded indium particles, Philos. Mag. A. (1995) 37-41. http://www.tandfonline.com/doi/abs/10.1080/01418619508236230 (accessed November 30, 2012).

[18] H. Rösner, G. Wilde, The impact of altered interface structures on the melting behaviour of embedded nanoparticles, Scr. Mater. 55 (2006) 119-122. doi:10.1016/j.scriptamat.2006.04.002.

[19] L.H. Zhang, E. Johnson, U. Dahmen, Observations of vacancy-controlled decay of elastic strain caused by phase transformation of small $\mathrm{Pb}$ inclusions in $\mathrm{Al}$, Acta Mater. 53 (2005) 3635-3642. doi:10.1016/j.actamat.2005.04.018.

[20] H. Rösner, J. Weissmüller, G. Wilde, HRTEM observation of misfit dislocations at non-faceted Al-Pb interfaces, Philos. Mag. Lett. 84 (2004) 673-683. doi:10.1080/09500830600939967.

[21] H. Rösner, C.T. Koch, G. Wilde, Strain mapping along Al-Pb interfaces, Acta Mater. 58 (2010) 162-172. doi:10.1016/j.actamat.2009.08.065. 
[22] A. Moros, H. Rösner, G. Wilde, Melting of faceted Pb nanoparticles at reduced latent heat, Scr. Mater. 65 (2011) 883-886. doi:10.1016/j.scriptamat.2011.07.056.

[23] M. McLean, M.S. Loveday, In-situ observations of the annealing of liquid lead inclusions entrained in an aluminium matrix, J. Mater. Sci. 9 (1974) 1104-1114.

[24] E. Johnson, S. Steenstrup, M. Levinsen, V. Prokofjev, V. Zhilin, U. Dahmen, Brownian motion of liquid lead inclusions along dislocations in aluminum, J. Mater. Sci. 40 (2005) 3115-3119. doi:10.1007/s10853-005-2672-6.

[25] T.B. Massalski, H. Okamoto, P.R. Subramanian, L. Kacprzak, Binary alloy phase diagrams, 2nd ed., ASM International, 1990.

[26] P. Schlossmacher, D.O. Klenov, B. Freitag, H.S. von Harrach, Enhanced Detection Sensitivity with a New Windowless XEDS System for AEM Based on Silicon Drift Detector Technology, Micros. Today. 18 (2010) 14-20. doi:10.1017/S1551929510000404.

[27] P. Schlossmacher, D.O. Klenov, B. Freitag, S. Von Harrach, A. Steinbach, Nanoscale Chemical Compositional Analysis with an Innovative S/TEM-EDX System, Microsc. Anal. Nanotechnol. Suppl. November (2010) 5-8.

[28] H. Wang, H. Zhu, Melting of Pb Nanocrystals Embedded in $\mathrm{Al}, \mathrm{Si}$, and $\mathrm{Cu}$ Matrices, Nanoscale Res. Lett. 10 (2015) 487. doi:10.1186/s11671-015-1196-5.

[29] S. Schmidt, Elektronenmikroskopische Untersuchungen zur Segregation von Ga an AlKorngrenzen, Max-Planck-Institut fuer Metallforschung, 2003.

[30] W.T. Kim, D.L. Zhang, B. Cantor, Nucleation of solidification in liquid droplets, Metall. Trans. A. 22 (1991) 2487-2501. doi:10.1007/BF02665015.

[31] H. Reichert, O. Klein, H. Dosch, M. Denk, V. Honkimäki, T. Lippmann, G. Reiter, Observation of five-fold local symmetry in liquid lead., Nature. 408 (2000) 839-41. doi: $10.1038 / 35048537$.

[32] S.H. Oh, Y. Kauffmann, C. Scheu, W.D. Kaplan, M. Rühle, Ordered liquid aluminum at the interface with sapphire., Science. 310 (2005) 661-663. doi:10.1126/science.1118611.

[33] S.E. Donnelly, R.C. Birtcher, C.W. Allen, I. Morrison, K. Furuya, M. Song, K. Mitsuishi, U. Dahmen, Ordering in a fluid inert gas confined by flat surfaces., Science. 296 (2002) 507-10. doi:10.1126/science.1068521. 


\section{Tables:}

\begin{tabular}{c|c|c|c|c} 
& $\mathrm{Al}_{99} \mathrm{~Pb}_{1}$ & $\mathrm{Al}_{98} \mathrm{Ga}_{1} \mathrm{~Pb}_{1}$ & $\mathrm{Al}_{96} \mathrm{Ga}_{3} \mathrm{~Pb}_{1}$ & $\mathrm{Al}_{93} \mathrm{Ga}_{6} \mathrm{~Pb}_{1}$ \\
\hline $\mathrm{Al}$ & $98.9 \pm 0.2$ & $97.8 \pm 0.2$ & $95.6 \pm 0.2$ & $93.4 \pm 0.7$ \\
\hline $\mathrm{Ga}$ & - & $1.1 \pm 0.1$ & $3.3 \pm 0.1$ & $5.8 \pm 0.7$ \\
\hline $\mathrm{Pb}$ & $1.1 \pm 0.1$ & $1.1 \pm 0.1$ & $1.0 \pm 0.1$ & $0.8 \pm 0.1$
\end{tabular}

Tab. 1: Chemical composition of the $\mathrm{Al}_{98} \mathrm{Ga}_{1} \mathrm{~Pb}_{1}, \mathrm{Al}_{96} \mathrm{Ga}_{3} \mathrm{~Pb}_{1}$ and $\mathrm{Al}_{93} \mathrm{Ga}_{6} \mathrm{~Pb}_{1}$ ribbons measured by energy dispersive X-ray application using an FEI Nova NanoSEM 230.

\begin{tabular}{l|c|c|c|c|c} 
& $\mathrm{T}_{\mathrm{m}, \text { onset }}[\mathrm{K}]$ & $\mathrm{T}_{\mathrm{m}, \text { end }}[\mathrm{K}]$ & $\mathrm{T}_{\mathrm{x}, \text { onset }}[\mathrm{K}]$ & $\Delta \mathrm{T}=\mathrm{T}_{\mathrm{m}}-\mathrm{T}_{\mathrm{x}}[\mathrm{K}]$ & $\Delta \mathrm{H}_{\mathrm{m}}[\mathrm{J} / \mathrm{g}]$ \\
\hline Bulk Pb & 600 & - & 594 & 6 & 23 \\
\hline $\mathrm{Al}_{99} \mathrm{~Pb}_{1}$ & 598 & 640 & 594 & 4 & $12.8 \pm 2.5$ \\
\hline $\mathrm{Al}_{98} \mathrm{Ga}_{1} \mathrm{~Pb}_{1}$ & 598 & 632 & 557 & 41 & $17.5 \pm 2.4$ \\
\hline $\mathrm{Al}_{96} \mathrm{Ga}_{3} \mathrm{~Pb}_{1}$ & 597 & 620 & 536 & 61 & $18.6 \pm 2.0$ \\
\hline $\mathrm{Al}_{93} \mathrm{Ga}_{6} \mathrm{~Pb}_{1}$ & 593 & 608 & 491 & 102 & $19.7 \pm 2.1$
\end{tabular}

Tab.2: Summary of the onset temperatures and the specific heat of fusion for all composites extracted from the DSC measurements. 


$\begin{array}{lccc}\text { Intensity } & \text { Intensity } & \text { Interface length } & \text { Ratio 1st } \\ \text { 1st layer } & \text { matrix } & {[\mathrm{nm}]} & \text { layer/matrix [\%] } \\ \text { [counts] } & \text { [counts] } & & \end{array}$

\begin{tabular}{|c|c|c|c|c|}
\hline $\begin{array}{c}\text { Matrix (yellow rectan } \\
\text { gle in Fig. 4) }\end{array}$ & - & $20180 \pm 460$ & - & - \\
\hline Interface $\mathrm{I}\{111\}$ & $20577 \pm 291$ & & 4.3 & $2.0 \pm 0.1$ \\
\hline Interface II $\{111\}$ & $20686 \pm 266$ & & 4.3 & $2.5 \pm 0.2$ \\
\hline Interface III $\{111\}$ & $20625 \pm 250$ & & 5.1 & $2.2 \pm 0.2$ \\
\hline Interface IV $\{111\}$ & $20722 \pm 295$ & & 5.0 & $2.7 \pm 0.2$ \\
\hline Interface V $\{100\}$ & $20430 \pm 305$ & & 2.4 & $1.2 \pm 0.1$ \\
\hline Interface VI $\{100\}$ & $20470 \pm 180$ & & 2.3 & $1.4 \pm 0.1$ \\
\hline
\end{tabular}

Tab. 3: Measured intensities and calculated intensity ratios from the high-resolution HAADFSTEM image shown in Fig. 5.

\begin{tabular}{c|c|c} 
composition & $\Theta\left[^{\circ}\right]$ & $\sigma_{\mathrm{L}}-\sigma_{\mathrm{S}}\left[\mathrm{mJ} / \mathrm{m}^{2}\right]$ \\
\hline $\mathrm{Al}_{99} \mathrm{~Pb}_{1}$ & 14 & 53 \\
\hline $\mathrm{Al}_{98} \mathrm{Ga}_{1} \mathrm{~Pb}_{1}$ & 21 & 51 \\
\hline $\mathrm{Al}_{96} \mathrm{Ga}_{3} \mathrm{~Pb}_{1}$ & 42 & 41 \\
\hline $\mathrm{Al}_{93} \mathrm{Ga}_{6} \mathrm{~Pb}_{1}$ & 72 & 17
\end{tabular}

Tab. 4. Calculated difference in interfacial energies of solid-Al/liquid-Pb determined from the DSC data using the approach of Kim et al. [30]. 

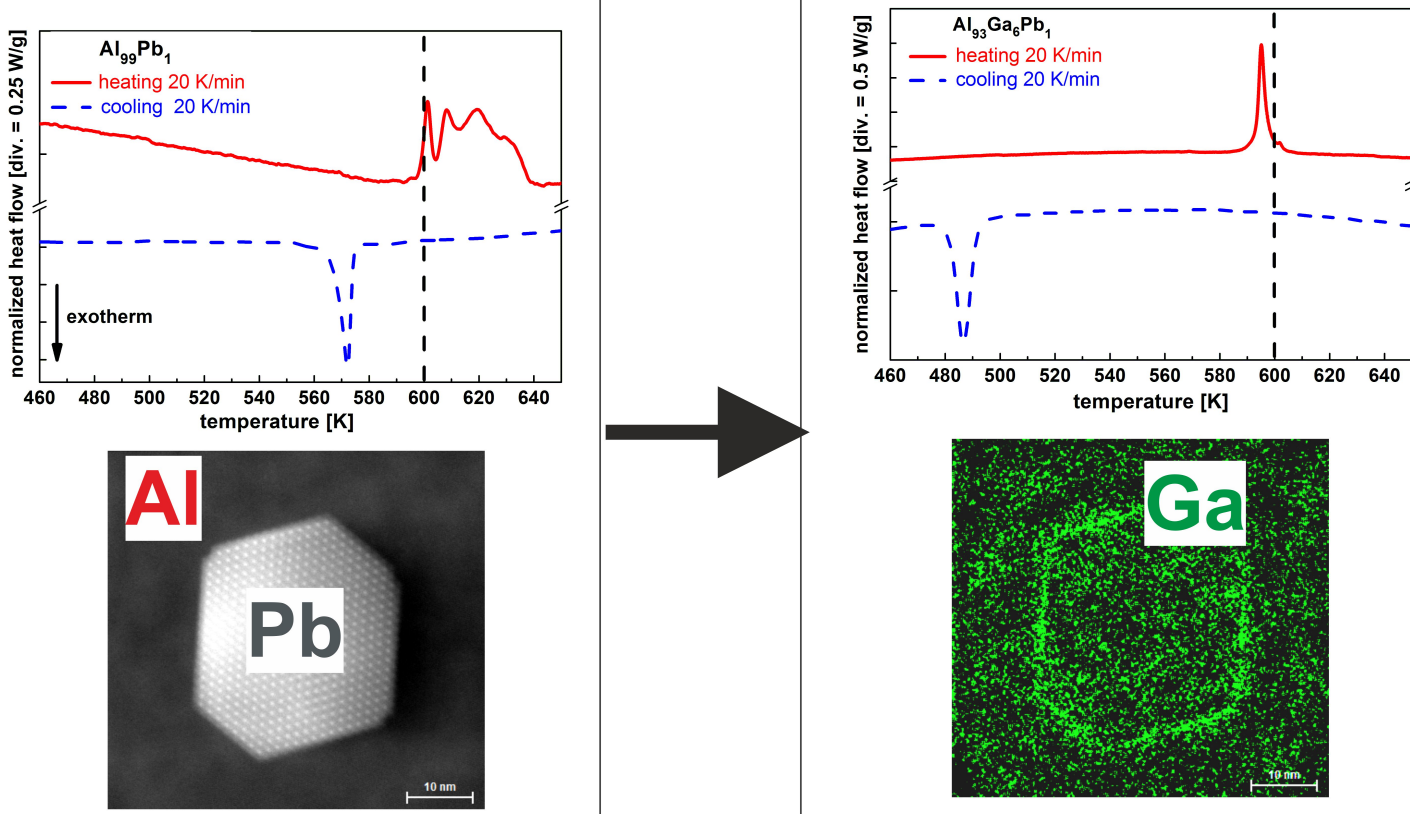